Orthopäde $2015 \cdot 44: 720-723$

DOI 10.1007/s00132-015-3143-7

Online publiziert: 24. Juli 2015

๑) Springer-Verlag Berlin Heidelberg 2015

CrossMark

\author{
P.-U.Tunn ${ }^{1}$ - M. Werner ${ }^{2}$ \\ ${ }^{1}$ Sarkomzentrum Berlin-Brandenburg, Zentrum für Orthopädie und Unfallchirurgie, \\ HELIOS Klinikum Berlin-Buch, Berlin, Deutschland \\ ${ }^{2}$ Institut für Gewebediagnostik Berlin, Medizinisches Versorgungszentrum, \\ HELIOS Klinikum Emil von Behring, Berlin, Deutschland
}

\title{
Osteosarkomatös dedifferenziertes Chondrosarkom
}

Zentrale und periphere ChondrosarDas zentrale Chondrosarkom ist nach dem Osteosarkom der zweithäufigste primär maligne Knochentumor und macht etwa $25 \%$ aller Knochensarkome aus [3]. Das Erkrankungsalter liegt vorwiegend in der 2. Lebenshälfte. Die Tumoren entstehen im Markraum, mehr als $50 \%$ der Tumoren sind stammnah lokalisiert. Es handelt sich in zwei Drittel der Fälle um G1und G2-Tumoren. G3-Chondrosarkome und dedifferenzierte Chondrosarkome sind wesentlich seltener [2, 3]. Im Gegensatz dazu sind periphere Chondrosarkome maligne knorpelbildende Tumoren, die sich aus einem vorbestehenden Osteochondrom entwickeln. Dabei kommt es zu einer erheblichen, autonomen $\mathrm{Zu}$ nahme des knorpeligen Gewebeanteils, dessen Ausdehnung den knöchernen Anteil des Osteochondroms weit übertreffen kann. Radiologisch sind Anteile des zugrunde liegenden Osteochondroms i. d. R. aber noch darstellbar. Es überwiegen niedrigmaligne Tumoren. kome werden nach wie vor chirurgisch behandelt. In den letzten Jahren hat sich gezeigt, dass hochdifferenzierte zentrale Chondrosarkome der Extremitäten intraläsional therapiert werden können, ohne die Prognose negativ zu beeinflussen [4, $9,12]$. Das trifft nicht für Chondrosarkome des Stammskeletts, unabhängig vom Grading, zu. Hier ist eine Heilung i. d. R. nur mit einer weiten Resektion (R0-Resektion) zu realisieren [8].

Das Chondrosarkom ist nach bisherigen Erkenntnissen weitgehend resistent gegenüber einer systemischen Chemotherapie [8], sodass diese vorwiegend in der palliativen Situation eingesetzt wird. Auch neue medikamentöse Therapiestrategien wie die Anwendung von Imatinib konnten bislang keinen prognostischen Vorteil im Rahmen einer Phase-II-Studie erbringen [6]. Eine mögliche Ausnahme könnte das dedifferenzierte Chondrosarkom darstellen. Patienten im Alter zwischen
40 und 65 Jahren können in das European-bone-over-forty-sarcoma-study(EURO-B.O.S.S.)-Protokoll eingeschlossen werden, in dem der Stellenwert der systemischen Chemotherapie geprüft wird. Da die Studie noch nicht geschlossen ist, kann diese Fragestellung derzeit nicht beantwortet werden.

Bei Patienten mit einem Morbus Ollier oder Mafucci-Syndrom beträgt das Risiko der Transformation in ein zentrales sekundäres Chondrosarkom bis etwa $40 \%$ [13]. Die maligne Transformation eines solitären Osteochondroms ist ein seltenes Phänomen [10]. Die Häufigkeit der Entstehung sekundärer peripherer Chondrosarkome bei der Osteochondromatose (Exostosenkrankheit) wird unterschiedlich eingeschätzt. Sie soll nach neueren Auswertungen 1-2\% betragen [11]. Die Transformation tritt vorwiegend nach dem 30. Lebensjahr ein. Da es sich überwiegend um G1- oder G2-Sarkome handelt, kann die Prognose güns-
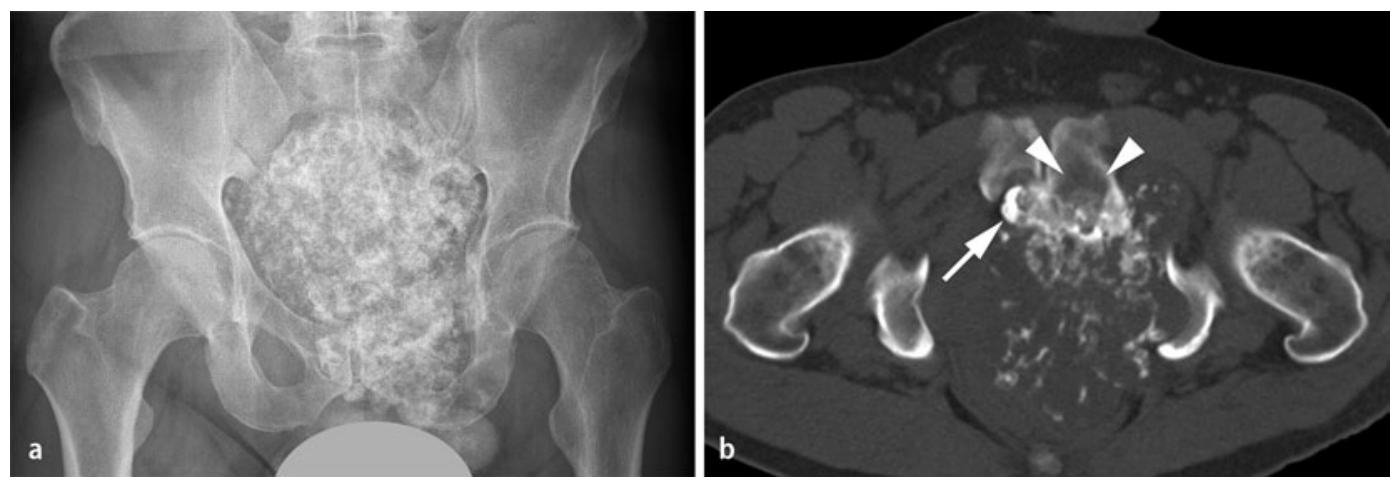

Abb. $1 \Delta$ Bildgebung bei Erstmanifestation. a) Beckenübersichtsaufnahme, großer exophytischer, fleckförmig verschatteter Tumor mit Ausgangspunkt von Ramus superior ossis pubis, der das kleine Becken weitgehend ausfüllt. b) Transversales Computertomogramm, Anteil des ursprünglichen Osteochondroms mit Insertion am Os pubis links, Impression in das Os pubis rechts (Pfeil), sehr ausgedehnte exophytische Anteile mit fleckförmigen Kalzifikationen im Binnenraum und an der Außenseite des kleinen Beckens ventral, durch Infiltration bedingte Aufhellung im Os pubis links (Pfeilspitzen) 

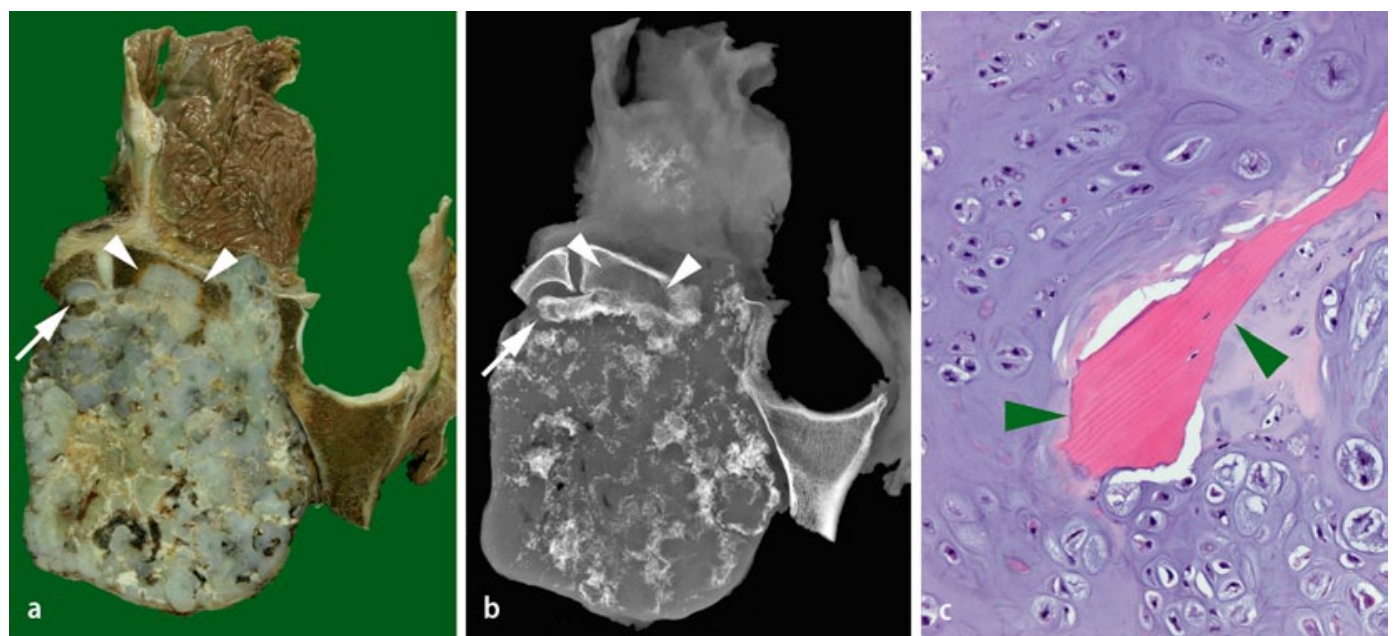

Abb. 2 A Hemipelvektomie, pathologisch-anatomische Aufarbeitung in strenger Korrelation zur computertomographischen Darstellung. Transversale Scheibe des Resektats, a lichtmikroskopische Aufnahme und b Kontaktradiographie dieser Scheibe, Acetabulum, Ramus superior ossis pubis links, Symphyse und parasymphysealer Anteil des Os pubis links mit Impression durch den Restanteil des ursprünglichen Osteochondroms (Pfeil), ausgedehnte exophytische, fleckförmig kalzifizierte Knorpelformationen im Beckenbinnenraum und intramuskulär ventral außen, Markrauminfiltration im Os pubis links (Pfeilspitzen); c) Histologisches Präparat des infiltrativen Tumoranteils, Tumorchondrozyten in basophiler chondroider Matrix, reaktionsloser Einschluss eines lamellären Spongiosatrabekels im Tumorgewebe (Pfeilspitzen)

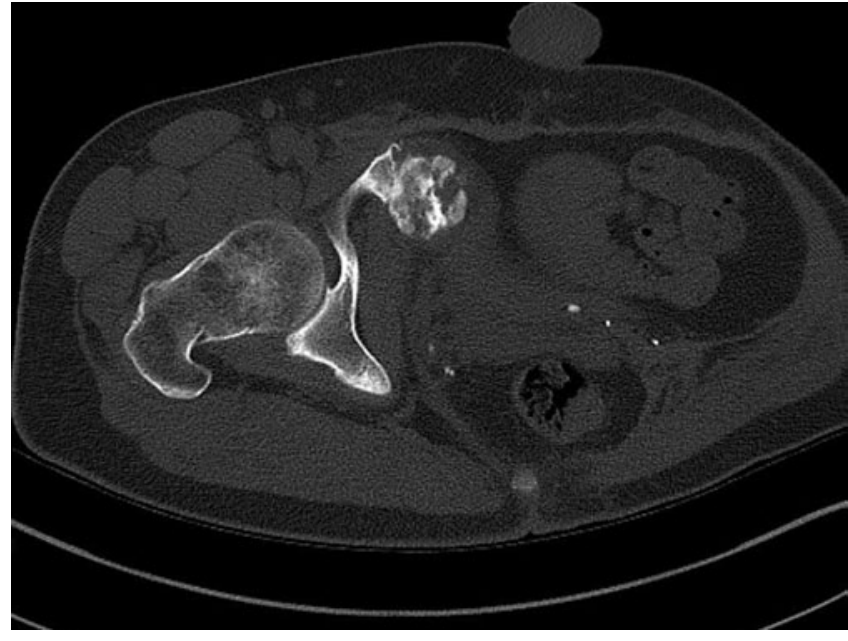

Abb. $3<$ BeckenComputertomographie des 2. Rezidivs. Zustand nach Hemipelvektomie links, exophytischer Rezidivtumor am Os pubis rechts

tig sein, wenn eine komplette Entfernung des exophytischen Tumors samt der Insertionsstelle zu realisieren ist.

Sowohl bei zentralen als bei peripheren Chondrosarkomen kann eine Dedifferenzierung eintreten. Dabei entwickelt sich neben dem ursprünglichen, meist niedrigmalignen Chondrosarkom ein neuer Tumoranteil, der feingeweblich eine andere Liniendifferenzierung aufweist (z. B. wie ein Osteosarkom oder Leiomyosarkom) oder gar keine Liniendifferenzierung erkennen lässt (wie ein undifferenziertes pleomorphes Sarkom). Die dedifferenzierten Anteile unterscheiden sich
Raumforderung, ausgehend vom Os pubis links dar (• Abb. 1).

Eine Computertomographie(CT)-gestützte Stanzbiopsie ergab die Diagnose eines hochdifferenzierten Chondrosarkoms. Unter Berücksichtigung der Bildgebung handelte es sich speziell um ein sekundäres peripheres Chondrosarkom auf dem Boden eines breitbasigen Osteochondroms, ausgehend vom Ramus superior ossis pubis, links. Hinweise für eine Fernmetastasierung ergaben sich nicht. Es resultierte im interdisziplinären Konsens unter Berücksichtigung der Tumorausdehnung die Empfehlung zur externen Hemipelvektomie links. Der Patient erklärte sich nach ausführlicher Aufklärung einverstanden, sodass der Eingriff im Oktober 2009 erfolgte. In der histopathologischen Aufarbeitung des Resektats bestätigte sich die Diagnose eines sekundären peripheren Chondrosarkoms. Der maligne transformierte Anteil war ganz überwiegend dem Malignitätsgrad 1, stellenweise, insbesondere in der Peripherie des Tumors, beginnend einem Grad 2 zuzuordnen (• Abb. 2). Für eine Dedifferenzierung ergab sich histopathologisch kein Anhalt. Die Tumorformel lautete: pT2 N0 M0 G1-2 R0, Stadium IB nach Union for International Cancer Control (UICC) 2003. Die Miktionsstörungen waren postoperativ komplett rückläufig. 
Im Verlauf wurde eine prothetische Versorgung vorgenommen; der Patient lernte, ohne Unterarmgehstützen sicher zu gehen.

Im Rahmen der Nachsorge wurde 3 Jahre nach dem Primäreingriff ein lokales Tumorrezidiv in der Fossa ischiorectalis links in einer Ausdehnung von $3 \times 4 \mathrm{~cm}$ diagnostiziert und bei fehlendem Anhalt für Fernmetastasen im November 2012 reseziert (lokal R0). Histologisch ergab sich ein hochdifferenziertes Chondrosarkom (G1) ohne dedifferenzierte Anteile. Die Nachsorge wurde fortgeführt.

Knapp ein Jahr später, im September 2013, wurde ein 2. Rezidiv am Os pubis der kontralateralen Seite in der CT des Beckens in einer Größe von $5 \times 6 \mathrm{~cm}$ nachgewiesen ( $\bullet$ Abb. 3) und reseziert. Es handelte sich anhand der makroskopischen und histologischen Befunde sowie unter Berücksichtigung der Bildgebung und der Vorbefunde um ein Lokalrezidiv eines sekundären peripheren Chondrosarkoms mit osteosarkomatöser Dedifferenzierung. Die Knorpelkappe hatte eine Dicke von $11 \mathrm{~mm}$. Der osteosarkomatöse Anteil war im Zentrum des Rezidivtumors lokalisiert (• Abb. 4). Metastasen waren im Rahmen des Stagings nicht zu detektieren. Aufgrund der histologischen Diagnose und unter Berücksichtigung des Lebensalters wurde die Einleitung einer adjuvanten systemischen Chemotherapie im EURO-B.O.S.S.-Protokoll empfohlen, vom Patienten jedoch abgelehnt. Der weitere Verlauf ist unbekannt.

\section{Diskussion}

Sekundäre periphere Chondrosarkome sind selten und hinsichtlich der Interpretation der bildgebenden und histopathologischen Diagnostik als auch der Therapie anspruchsvoll. Sie weisen meist ein langsames Tumorwachstum auf und werden insbesondere im Becken erst spät diagnostiziert, sodass sich nicht selten Patienten mit lokal fortgeschrittenen Tumoren vorstellen. Die Symptome sind häufig unspezifisch und reichen über ein Völlegefühl, einer langsam progredienten Schwellung bis hin zu Miktions- und Defäkationsbeschwerden aufgrund eines meist verdrängend wachsenden Tumors. Ein infiltratives Wachstum in die Orga- ne des kleinen Beckens kann im Verlauf nicht ausgeschlossen werden. Das Erkrankungsalter liegt meist 10-20 Jahre unter dem von Patienten mit primärem Chondrosarkom [1].

Hinsichtlich der bildgebenden Diagnostik haben sich, neben der nativröntgenologischen Untersuchung, die CT und die Magnetresonanztomographie (MRT) bewährt. In der CT kann die Matrix, in der MRT die intramedulläre Ausdehnung des Tumors und die Beziehung zu den Nachbarstrukturen beurteilt werden.

Sekundäre periphere Chondrosarkome sind meist gut bis intermediär differenziert. Sie haben nach der kompletten chirurgischen Entfernung eine gute Prognose. In einer großen retrospektiven Studie zeigten Ahmed et al. [1] anhand von 107 Patienten, dass die 5- und 10-JahresLokalrezidivrate bei 15,9 und 17,5\% liegt. Fernmetastasen treten in weniger als 5\% der Fälle auf.

Dedifferenzierte Chondrosarkome (zentral bzw. peripher) sind noch seltener. Die Prognose scheint schlechter zu sein. Da es sich bei den publizierten Daten meist um Fallberichte handelt, werden prognostisch relevante Aussagen weitgehend vermisst [6]. Die R0-Resektion hat nach heutigem Wissensstand den wichtigsten prognostischen Stellenwert [2, 3, $8,11,14]$.

\section{Fazit für die Praxis}

- Periphere sekundäre Chondrosarkome sind selten. Sie entstehen auf dem Boden eines Osteochondroms, die Ätiologie ist nicht bekannt.

- Da es sich vorwiegend um hochdifferenzierte Tumoren handelt, ist die Prognose nach erfolgter R0-Resektion als günstig einzuschätzen.

- Eine tumorspezifische Nachsorge sollte dennoch erfolgen, um ein Lokalrezidiv frühzeitig erkennen und behandeln zu können.

- In dem sehr seltenen Fall einer osteosarkomatösen Dedifferenzierung in einem Rezidiv eines peripheren sekundären Chondrosarkoms besteht eine deutlich erhöhte Metastasierungswahrscheinlichkeit. Somit ist neben der operativen Therapie des Lokalrezidivs auch die Empfehlung
Orthopäde 2015 • 44:720-723

DOI 10.1007/s00132-015-3143-7

○ Springer-Verlag Berlin Heidelberg 2015

\section{P.-U. Tunn $\cdot$ M. Werner}

\section{Osteosarkomatös dedifferenziertes Chondrosarkom}

\section{Zusammenfassung}

Ein sekundäres peripheres Chondrosarkom (SPC) auf dem Boden einer kartilaginären Exostose ist selten, aber mehrfach beschrieben. Die Entwicklung eines osteosarkomatös dedifferenzierten Chondrosarkoms in einem Rezidiv eines SPC ist eine Rarität. Anhand einer Kasuistik wird dieses Phänomen bei einem 46-jährigen Patienten demonstriert und diskutiert.

\section{Schlüsselwörter}

Neoplastische Zelltransformationen · Zweite primäre Neoplasmen · Lokalrezidiv · Grading . Exostose

\section{Osteosarcomatous \\ dedifferentiated chondrosarcoma}

\section{Abstract}

A secondary peripheral chondrosarcoma (SPC) evolving from an osteochondroma is rare, but it has been described several times. The development of an osteosarcomatous dedifferentiated chondrosarcoma in a locally recurrent SPC is extremely rare. The following case report of a 46-year-old man demonstrates and discusses this phenomenon.

\section{Keywords}

Neoplastic cell transformation - Second primary neoplasms - Local recurrence . Neoplasm grading $\cdot$ Exostosis

zur Durchführung einer systemischen Chemotherapie in Anlehnung an die Osteosarkom-Studienprotokolle gegeben.

\section{Korrespondenzadresse}

PD Dr. P.-U. Tunn

Sarkomzentrum Berlin-Brandenburg Zentrum für Orthopädie und Unfallchirurgie, HELIOS Klinikum Berlin-Buch

Schwanebecker Chaussee 50, 13125 Berlin per-ulf.tunn@helios-kliniken.de 

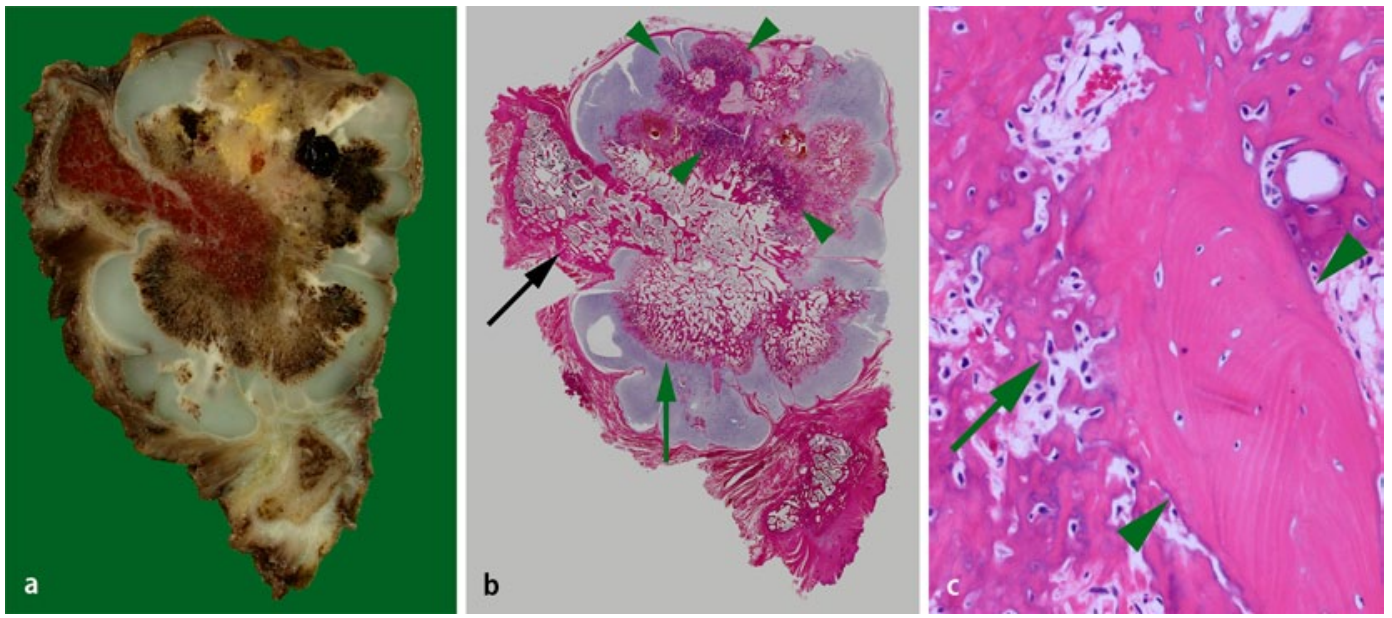

Abb. $4 \Delta$ Transversale Scheibe des Resektats. a Lichtmikroskopische Aufnahme und b histologischer Großschnitt der gesamten Scheibe mit Os pubis (schwarzer Pfeil), osteochondromatösem Anteil mit Knorpelkappe, spongiösem Knochengewebe und einem wachstumszonenartigen Übergangsbereich (grüner Pfeil), Entwicklung eines Osteosarkoms im Inneren unterhalb der Knorpelkappe (Pfeilspitzen); c Vergrößerung osteosarkomatösen Anteils von b, kleine Tumorzellen, die sich in gitterförmiges Osteoid einmauern (Pfeil), permeativer Einschluss eines lamellären Knochentrabekels (Pfeilspitzen)

\section{Einhaltung ethischer Richtlinien}

Interessenkonflikt. P.-U. Tunn und Dr. M. Werner geben an, dass kein Interessenkonflikt besteht.

Dieser Beitrag beinhaltet keine Studien an Menschen oder Tieren.

\section{Literatur}

1. Ahmed AR, Tan TS, Unni KK, Collins MS, Wenger $\mathrm{DE}$, Sim FH (2003) Secondary chondrosarcoma in osteochondroma: report of 107 patients. Clin Orthop Relat Res 411:193-206

2. Altay M, Bayrakci K, Yildiz Y, Erekul S, Saglik Y (2007) Secondary chondrosarcoma in cartilage bone tumors: report of 32 patients. J Orthop Sci 12(5):415-423

3. Andreou D, Ruppin S, Fehlberg S, Pink D, Werner M, Tunn PU (2011) Survival and prognostic factors in chondrosarcoma. Acta Orthop 82(6):749-755

4. Campanacci DA, Scoccianti G, Franchi A, Roselli G, Beltrami G, Ippolito M et al (2013) Surgical treatment of central grade 1 chondrosarcoma of the appendicular skeleton. J Orthop Traumatol 14(2):101-107

5. Franchi A, Baroni G, Sardi I, Giunti L, Capanna R, Campanacci D (2012) Dedifferentiated peripheral chondrosarcoma: a clinicopathologic, immunohistochemical, and molecular analysis of four cases. Virchows Arch 460(3):335-342

6. Grignani G, Palmerini E, Stacchiotti S, Boglione A, Ferraresi V, Frustaci $S$ et al (2011) A phase 2 trial of imatinib mesylate in patients with recurrent nonresectable chondrosarcomas expressing plateletderived growth factor receptor- $\alpha$ or $-\beta$ : an Italian Sarcoma Group study. Cancer 117(4):826-831

7. Italiano A, Mir O, Cioffi A, Palmerini E, PipernoNeumann S, Perrin C et al (2013) Advanced chondrosarcomas: role of chemotherapy and survival. Ann Oncol 24(11):2916-2922

8. Mavrogenis AF, Angelini A, Drago G, Merlino B, Ruggieri $P$ (2013) Survival analysis of patients with chondrosarcomas of the pelvis. J Surg Oncol 108(1):19-27
9. Meftah M, Schult P, Henshaw RM (2013) Long-term results of intralesional curettage and cryosurgery for treatment of low-grade chondrosarcoma. J Bone Joint Surg Am 95(15):1358-1364

10. Nystrom LM, DeYoung BR, Morcuende JA (2013) Secondary chondrosarcoma of the pelvis arising from a solitary exostosis in an 11-year-old patient: a case report with 5-year follow-up. lowa Orthop J 33:213-216

11. Sonne-Holm E, Wong C, Sonne-Holm S (2014) Multiple cartilaginous exostoses and development of chondrosarcomas - a systematic review. Dan Med J 61(9):A4895

12. Verdegaal SH, Brouwers HF, van Zwet EW, Hogendoorn PC, Taminiau AH (2012) Low-grade chondrosarcoma of long bones treated with intralesional curettage followed by application of phenol, ethanol, and bone-grafting. J Bone Joint Surg Am 94(13):1201-1207

13. Verdegaal SH, Bovée JV, Pansuriya TC, Grimer RJ, Ozger H, Jutte PC et al (2011) Incidence, predictive factors, and prognosis of chondrosarcoma in patients with ollier disease and maffucci syndrome: an international multicenter study of 161 patients. Oncologist 16(12):1771-1779

14. Wuisman PI, Jutte PC, Ozaki T (1997) Secondary chondrosarcoma in osteochondromas. Medullary extension in 15 of 45 cases. Acta Orthop Scand 68(4):396-400 